\title{
Economie et politique de la chasse. Application au cas français
}

Economics and policy of hunting: application to the French case

\section{Philippe Le Goffe}

\section{(2) OpenEdition}

\section{Journals}

\section{Édition électronique}

URL : http://journals.openedition.org/economierurale/3329

DOI : 10.4000/economierurale.3329

ISSN : 2105-2581

\section{Éditeur}

Société Française d'Économie Rurale (SFER)

\section{Édition imprimée}

Date de publication : 1 mars 2012

Pagination : 11-23

ISSN : 0013-0559

\section{Référence électronique}

Philippe Le Goffe, «Economie et politique de la chasse. Application au cas français », Économie rurale [En ligne], 327-328 | janvier-mars 2012, mis en ligne le 01 mars 2014, consulté le 19 avril 2019. URL: http://journals.openedition.org/economierurale/3329; DOI : 10.4000/economierurale.3329 


\section{Économie et politique de la chasse Application au cas français}

Philippe LE GOFFE • UMR Structures et Marchés agricoles, Ressources et Territoires, SMART, Agrocampus Ouest, INRA, Rennes

philippe.legoffe@agrocampus-ouest.fr

\section{Introduction}

B ien que la chasse soit marchandisée à des degrés divers, selon qu'elle est privée, domaniale ou associative, des politiques publiques sont nécessaires pour réguler la chasse elle-même ou ses interactions avec la société. L'objectif de l'article ${ }^{1}$ est de proposer une lecture économique originale des questions posées par l'exercice de la chasse en France, et d'en déduire des recommandations qui pourraient permettre d'améliorer les politiques cynégétiques.

Les enjeux cynégétiques sont très diversifiés. L'importance de la chasse associative par rapport aux chasses privées explique que nous ayons l'effectif de chasseurs le plus élevé d'Europe, malgré une baisse régulière. Cette tradition d'accès non restrictif aux territoires de chasse dans de nombreuses régions françaises a l'intérêt de démocratiser la chasse, mais a pu aussi être à l'origine d'une pression de chasse élevée, tant en prélèvement qu'en fréquentation. Dans leur atlas de la biodiversité de la faune sauvage, Vallance et al. (2008) indiquent que des prélèvements mal maîtrisés ont constitué un des facteurs du déclin des populations de perdrix grises et rouges, de lapins de garenne et de lièvres. Par ailleurs, à propos des facteurs de variation de la valeur du droit de chasse, Fischer (1999) évoque l'effectif trop impor-

1. Cet article est issu d'une communication plus large présentée au colloque SFER «Chasse, territoires et développement durable : outils d'analyse, enjeux et perspectives » qui s'est tenu du 25 au 27 mars 2009 à Clermont-Ferrand. L'auteur remercie le lecteur anonyme dont les commentaires ont permis d'améliorer l'article. tant des chasseurs dans les Associations communales de chasse agréées (ACCA). Cependant, le mauvais état de certaines populations, notamment de petit gibier de plaine, est aussi dû aux modifications de leur habitat par l'agriculture. Reitz (2006) considère que l'évolution des milieux agricoles est la cause essentielle de la division par trois des tableaux de chasse nationaux en perdrix grise, lapin et lièvre entre 1974 et 1998. L'intensification de l'agriculture, mais aussi la déprise agricole et la fermeture du milieu qui en résulte, sont mis en cause, notamment dans la régression des populations de lapins de garenne et de perdrix rouges (Vallance et al., op. cit.). De la même façon, la régression des zones humides a pu affecter de manière importante les effectifs de canard de surface, comme dans le marais poitevin où $50 \%$ des prairies humides ont été drainées (Vallance et al., op. cit.). À l'opposé, la prolifération du gros gibier, qui constitue aujourd'hui la base de la chasse française, est responsable de dégâts agricoles et forestiers qui grèvent le budget des fédérations de chasse. Enfin, la chasse peut aussi générer des conflits d'usage avec la société en général.

L'article comporte trois sections. La première section propose une explication des inefficacités décrites dans le constat précédent à partir de la structure des droits de propriété et des concepts de bien collectif et d'externalité. La deuxième section applique à la chasse les théories économiques de la gestion optimale des ressources et de l'environnement. La troisième section utilise les concepts des deux sections précédentes pour tenter une analyse économique 
des politiques cynégétiques actuelles et présenter quelques propositions. Les questions relatives à la coopération collective entre les acteurs et aux coûts de transaction sont discutées en conclusion.

\section{Droits de propriété Biens collectifs et externalités}

En s'appuyant sur le droit romain qui classe les biens en fonction des relations d'appropriation, Landelle (2005) s'interroge sur le statut juridique qui convient à la faune sauvage. Il distingue notamment, parmi les res extra commercium ou choses hors commerce, les res communes, « choses qui n'appartiennent à personne et dont l'usage est commun à tous », et parmi les res in commercio que l'on peut vendre, les res in patrimonio qui font l'objet de droits privatifs. Ces deux statuts opposés lui paraissent incompatibles avec la nécessaire gestion collective d'un patrimoine commun, car elle n'est pas prévue dans le premier cas et passe après les bénéfices privés dans le deuxième.

\section{Biens collectifs}

Pour l'économiste, les atteintes aux ressources en gibier et à leurs habitats s'expliquent en partie par le fait que, face aux signaux du marché qui orientent les activités économiques, on a ici souvent affaire à des biens collectifs pour lesquels les agents ne reçoivent pas de signaux, dans la mesure où ces biens sont hors marché. L'absence de droits de propriété qui caractérise ces biens rend impossible l'exclusion, condition nécessaire à toute transaction marchande. Autrement dit, les agents n'ont pas d'incitations économiques à épargner les ressources et à protéger ou produire leurs habitats. D'un point de vue économique, on peut dire qu'il n'y a pas de coordination marchande entre la demande des chasseurs et l'offre de chasse, qui existe pourtant et émane des propriétaires privés et publics, forestiers et agricoles. L'objet des politiques de la chasse est de pallier ces défaillances du marché.

On peut diviser ces biens collectifs en plusieurs catégories, selon la classification économique classique.

- On trouve d'abord de véritables ressources communes, non exclusives, mais rivales en ce sens que le prélèvement d'un chasseur diminue la jouissance des autres chasseurs en puisant dans le stock. Quand elles sont en accès libre, ces ressources sont sujettes au dilemme du prisonnier et à la « tragédie des communs » qui en résulte, c'est-à-dire que la stratégie individuelle dominante, qui consiste à prélever tant qu'on en tire de la satisfaction, conduit à la surexploitation des ressources. Avec ce type de ressources, ce mécanisme est d'ailleurs à l'œuvre même lorsque le droit de chasse est privatif. Les stocks d'oiseaux migrateurs, qu'il s'agisse de gibier d'eau ou de migrateurs terrestres appelés « oiseaux de passage », constituent l'essentiel de cette catégorie.

- On trouve ensuite des biens publics non exclusifs et non rivaux, encore qu'ils puissent être plus ou moins purs (existence d'encombrement notamment). Les zones de reproduction des oiseaux migrateurs, souvent situées à l'étranger, ainsi que les domaines communaux et de l'État font partie de cette catégorie. On notera ici que les forêts domaniales gérées par l'Office national des forêts (ONF) font l'objet d'adjudications et deviennent dans ce cas des « biens clubs », pour lesquels l'exclusion est possible. Dans une certaine mesure, les dégâts provoqués par le gros gibier appartiennent aussi à cette catégorie, à la différence qu'il s'agit de maux publics ${ }^{2}$. Ici, c'est la divagation des animaux sauvages et l'impossibilité d'identifier avec certitude leur fonds d'origine qui donnent un caractère

2. Plus largement, les dangers pour les biens et les personnes occasionnés par la chasse peuvent également être qualifiés de maux publics. 
public aux dégâts aux cultures et aux collisions automobiles, bien que les gibiers concernés soient sédentaires. En revanche, les dégâts forestiers des cervidés sont considérés comme des biens privés quand le propriétaire conserve le droit de chasse, dans la mesure où ce dernier est censé arbitrer entre les bénéfices de la chasse et la régulation des populations et des dégâts. C'est d'ailleurs pourquoi ces dégâts privés ne donnent pas droit à une indemnisation collective, alors que cela est possible dans le cas des ACCA et dans les départements d'Alsace-Moselle, où le droit de chasse est administré par la commune (Charlez, 2008a).

\section{- Enfin, il y a le cas des territoires gérés par} la chasse associative, très représentée en France puisqu'il existe en moyenne plus de deux sociétés de chasse par commune, soit 80000 en tout. Cette forme d'organisation présente l'intérêt d'offrir un accès équitable à l'activité de chasse ${ }^{3}$. Le droit de chasse est abandonné par le propriétaire au profit des sociétés de chasse, par le biais d'un bail de chasse, à un prix souvent modique (Fischer, $o p$. cit.). Ce transfert de droits de propriété modifie le statut du droit de chasse : bien privé lié au droit de propriété, il devient un bien public (territoires de chasse) ou une ressource commune (gibier), catégories exposées aux défaillances du marché. Dans le cas particulier des ACCA, qui concerne un tiers des communes françaises ${ }^{4}$, ce transfert est organisé par la loi Verdeille du 10 juillet 1964 (Charlez, 2003 et 2008b). Cette loi oblige les propriétaires à apporter gratuitement leur droit de chasse à l'ACCA si la

3. Cette tradition devrait être renforcée. La proposition de loi relative à l'amélioration et à la simplification du droit de la chasse prévoit de diminuer et d'harmoniser le coût des timbres et cotisations et de faciliter l'accueil des chasseurs sur les territoires de chasse, via des cartes temporaires.

4. Les ACCA sont obligatoires dans un tiers des départements français. Ailleurs, il faut l'accord préalable d'au moins $60 \%$ des propriétaires représentant plus de $60 \%$ du territoire de la commune. surface de leur propriété est inférieure à un seuil variant entre 20 et 60 hectares ${ }^{5}$. À la suite de contestations devant les juridictions européennes, la loi relative à la chasse du 26 juillet 2000 permet désormais à un propriétaire de refuser cet apport obligatoire au nom de ses convictions personnelles (opposition de conscience ou philosophique), à condition de renoncer à l'exercice du droit de chasse. Outre l'équité dans l'accès, l'objectif des ACCA est aussi de favoriser une gestion cynégétique rationnelle, en évitant les pratiques anarchiques. Les atteintes aux ressources et aux habitats citées en introduction montrent néanmoins qu'il peut exister des contradictions entre cet objectif de gestion cynégétique rationnelle et les défaillances du marché liées à l'abandon des droits de propriété, si des mesures de gestion collective ne sont pas prises.

L'impossibilité de commercialiser les biens collectifs de la sphère cynégétique, en raison de l'absence ou de l'abandon des droits de propriété, produit des situations d'externalités, c'est-à-dire des interactions positives ou négatives entre agents économiques, sans qu'il y ait versement de compensations monétaires. Ces situations sont caractérisées par l'absence d'incitations et par des pertes de valeur (inefficacité économique). L'activité chasse est le lieu d'externalités entre chasseurs, entre chasseurs et agriculteurs et plus largement entre chasseurs et le reste de la société.

\section{Externalités entre chasseurs}

Même si la chasse est une activité sociale où la convivialité est importante, les chasseurs se concurrencent à travers la pression qu'ils

\footnotetext{
5. Cette disposition existe également dans le régime de droit local des communes d'Alsace-Moselle, à la différence que les territoires ainsi regroupés sont ensuite mis en adjudication publique, ce qui leur confère un statut de bien club comparable à celui des forêts gérées par l'ONF. Ceci explique que la valeur du droit de chasse y soit plus élevée que dans les ACCA (Fischer, 1999).
} 
exercent sur les stocks de gibier et sur l'espace, dans la mesure où chacun préfère chasser dans une population abondante, avec beaucoup d'espace vital. Ces phénomènes hors marché sont qualifiés d'externalités de stock et d'encombrement. Quand l'accès au territoire de chasse et à la ressource est libre et gratuit, les chasseurs ne sont pas incités à restreindre leur prélèvement ou leur fréquentation, ce qui conduit à la surexploitation des ressources et de l'espace, et en conséquence à la réduction du bien-être collectif des chasseurs. On retrouve ici la «tragédie des communs ».

\section{Entre chasseurs et agriculteurs}

La chasse est également le lieu de nombreuses externalités environnementales, qui le plus souvent font interagir les chasseurs et les agriculteurs. C'est ainsi que les dégâts du gibier aux cultures sont assimilables à une externalité de pollution. Ici, le caractère collectif des dégâts fait qu'il est difficile de les imputer au fonds d'origine des animaux. Ceci explique que les chasseurs ne soient pas incités à réduire les populations et par suite les dégâts, qui sont donc excessifs au regard des bénéfices que la chasse procure, dans les situations où les chasseurs ont collectivement réussi à limiter leurs prélèvements pour augmenter les populations. C'est ce mécanisme qui a conduit à l'augmentation constante des dépenses d'indemnisation des dégâts de gibier au cours des vingt dernières années, jusqu'à mettre en péril les caisses d'indemnisation.

\section{Agriculture, biodiversité et chasse}

Les écosystèmes agricoles traditionnels ont sélectionné une biodiversité et notamment une faune cynégétique particulières (céréales et perdrix, prairies humides et gibier d'eau, etc.). Au cours des dernières décennies, les signaux du marché ont incité les agriculteurs à intensifier, voire à abandonner l'agriculture, deux phénomènes qui mettent en danger les équilibres agro-cynégétiques. Autrement dit, l'agriculture peut produire de la biodiversité, mais cette production a un coût. Le problème est que, dans le cas où les droits de propriété ont été abandonnés, c'està-dire dans le système associatif et tout particulièrement les ACCA, les agriculteurs n’ont plus la possibilité de répercuter ces coûts sur les usagers, qui profitent de ces biens collectifs en « passager clandestin ». Dans cette situation d'externalité, les agriculteurs ne sont plus incités à faire les améliorations environnementales favorables au gibier, même si les chasseurs y attachent un prix qui dépasse leur coût pour les agriculteurs. Ceci explique la dégradation continue des habitats du petit gibier de plaine et du gibier d'eau, plus ou moins marquée en fonction de l'évolution des systèmes de production agricole. Cet inconvénient devrait être atténué en régime de droit local d'Alsace-Moselle, dans la mesure où les revenus de la location des droits de chasse sont répartis par la commune entre les propriétaires (Fischer, 1999).

Un fait récent illustre les difficultés liées à cette externalité inhérente au système associatif. Dans son livre blanc sur le bilan de santé de la PAC, la FNC (2008) demande à la Commission européenne qu'un dispositif spécifique relatif à la biodiversité soit prévu dans le premier pilier de la PAC, avec application obligatoire à l'ensemble des espaces agricoles. La jachère obligatoire avait permis de développer des jachères « environnement-faune sauvage » subventionnées, mais facultatives. Face à la suppression de la jachère obligatoire, la FNC propose d'imposer à toute exploitation un DPU biodiversité, dans lequel $5 \%$ de la SAU serait consacré à la biodiversité en contrepartie du versement du DPU ${ }^{6}$ (haies,

6. Le Droit à paiement unique représente environ $300 €$ de l'hectare. Le projet de réforme de 2013 va dans le sens de la proposition de la FNC. En plus des paiements de base, il est prévu des paiements verts, notamment en faveur de réservoirs écologiques, qui représenteraient $30 \%$ des paiements directs. 
talus, mares, couverts faunistiques). En outre la FNC propose d'intégrer la biodiversité dans la conditionnalité, notamment en exigeant une plus grande diversité d'assolement dans les $\mathrm{BCAE}^{7}$, et se déclare favorable à l'article $69^{8}$ qui permettrait de réaffecter des aides du premier pilier à une agriculture favorable à environnement. Ces propositions ont été mal reçues par la FNSEA ${ }^{9}$. Quel que soit l'intérêt général de ces propositions, cette réaction peut se comprendre dans la mesure où les coûts correspondants seraient entièrement supportés par les agriculteurs, alors que les chasseurs en bénéficieraient gratuitement en raison de l'externalité.

\section{La gestion optimale des ressources et de l'environnement}

Dans la théorie économique, la gestion optimale des ressources et de l'environnement fait référence à la notion d'optimum de Pareto. Il s'agit de l'état de l'économie le plus efficace possible qui, dans une vision simplifiée, maximise la valeur collective calculée comme la somme algébrique des bénéfices et des coûts attachés aux actifs en question. C'est pourquoi rechercher l'optimum revient à comparer des bénéfices et des coûts marginaux, c'est-à-dire procéder à une analyse coûts-bénéfices.

Dans le cas des ressources naturelles, cet arbitrage économique est couplé à la dynamique de croissance de la ressource, d'où

7. Bonnes conditions agricoles et environnementales.

8. L'article 69, inscrit dans la réforme de la PAC de 2003 , permet de rediriger des fonds du premier pilier (jusqu'à $10 \%$ ) vers des «paiements supplémentaires » à des « types particuliers d'agriculture qui sont importants pour la protection ou l'amélioration de l'environnement ou pour l'amélioration de la qualité ».

9. Les réactions du président et d'un membre du bureau de la FNSEA sont rapportées dans un entrefilet paru dans Agra Presse Hebdo du 2 juin 2008, intitulé «L'agacement de la FNSEA ». l'appellation de modèles bioéconomiques. Ces modèles ont d'abord été développés pour les ressources exploitées commercialement et notamment pour les pêcheries. Le stock optimal est le stock stationnaire qui maximise le profit collectif de la pêcherie, égal à la recette diminuée du coût de l'effort de pêche. Ce coût augmente avec l'effort, mais diminue quand le stock est abondant, ce qui est à l'origine d'une externalité de stock, dans la mesure où le prélèvement des uns augmente le coût des autres. Alors que les premiers modèles étaient statiques (Gordon, 1954), les travaux plus récents ont privilégié des modèles dynamiques qui maximisent le profit sur un horizon intertemporel (Clark, 1990). Bien que beaucoup moins nombreux, les modèles bioéconomiques sur la chasse s'inspirent de ces travaux sur les pêcheries. Les modèles recensés dans la littérature traitent surtout de l'arbitrage entre les bénéfices procurés par la chasse et les dommages provoqués par le gibier, dans le but de déboucher sur le contrôle des populations (Zivin et al., 2000 ; Rondeau, 2001 ; Horan et Bulte, 2004 ; Skonhoft et Olaussen, 2005 ; Rakotoarison et Point, 2009 ; Rakotoarison et al., 2009 ; Ropars-Collet et Le Goffe, 2009). Rares sont les travaux théoriques qui s'intéressent à la gestion de l'encombrement ou de l'environnement.

Cette section reprend l'approche développée dans la littérature sur la chasse en introduisant comme Ropars-Collet et Le Goffe (op. cit.) une externalité de stock (à la manière des pêcheries) et en élargissant l'analyse aux questions d'encombrement et d'environnement, évoquées dans la section précédente. À la différence des travaux cités en référence, comme par exemple ceux présentés au colloque de la SFER en 2009, l'objectif n'est pas d'estimer empiriquement les populations et les efforts optimaux. Nous avons choisi à dessein de présenter les modèles de façon non technique, en mettant l'accent sur les arbitrages issus des conditions d'optimalité et l'utilisation qui peut en 
être faite pour calibrer des incitations. L'objectif est ici de préparer la section suivante, qui propose une réflexion sur les politiques de la chasse. Les programmes exposés dans la suite maximisent la valeur collective sur une période, par souci de simplicité, alors qu'il conviendrait de considérer la somme des valeurs collectives actualisées sur un horizon infini, c'est-à-dire des programmes dynamiques et non pas statiques. Cette option incomplète, qui donne les arbitrages mais pas la dynamique des équilibres, a également été retenue par Skonhoft et Olaussen (op. cit.) notamment.

S'agissant de la chasse, les bénéfices correspondent à la valeur que lui attribuent les chasseurs, c'est-à-dire au Consentement à payer (CAP) pour pratiquer l'activité. On fait l'hypothèse que le CAP marginal diminue avec la quantité de gibier ou de sorties consommée par le chasseur, mais augmente avec le stock global (externalité de stock) et les améliorations environnementales et diminue avec la fréquentation globale (externalité d'encombrement). Cela correspond à la demande inverse de chasse, fonction décroissante qui met en relation le prix par pièce de gibier (prix du bracelet par exemple) ou par sortie (prix de la carte à la journée) et les quantités consommées. Ces hypothèses traduisent, d'une part la satiété progressive du chasseur au fur et à mesure qu'il consomme de l'activité chasse et, d'autre part, ses préférences pour des populations abondantes et des territoires propices au gibier, peu encombrés par les autres chasseurs. En toute rigueur, il faudrait soustraire le coût de l'effort de chasse de ces bénéfices. Horan et Bulte (op. cit.) notent que le CAP marginal, net du coût marginal de l'effort de chasse, peut être positif ou négatif. Dans ce dernier cas, l'excédent de coût peut être assimilé à un consentement à recevoir. Cette situation peut se rencontrer quand la chasse est utilisée pour réguler certaines espèces proliférantes ou peu valorisées par les chasseurs. Il faudrait alors envisager de rémunérer les chasseurs, comme on le fait pour les professionnels du piégeage.

\section{Optimum des chasseurs}

En situation d'accès libre et gratuit (pas de bracelet payant) et en l'absence de contrôle des prélèvements, le chasseur prélève du gibier tant qu'il en retire du bien-être, c'està-dire jusqu'à l'annulation de son CAP pour la dernière pièce de gibier (CAP marginal). C'est cet équilibre d'accès libre qui peut être qualifié de «tragédie des communs ». Alors qu'à l'équilibre le chasseur considère que son prélèvement est trop faible pour avoir un impact sur le stock, le programme optimal qui maximise le CAP collectif, somme des CAP des chasseurs, intègre le fait que le prélèvement diminue le stock et par suite le bien-être des chasseurs. À l'optimum, le prélèvement global doit être amené jusqu' au niveau où le CAP marginal est égal à la perte de bien-être imposée à tous les chasseurs du fait de la diminution marginale du stock (externalité marginale de stock). Le prélèvement optimal est plus faible que le prélèvement qui annule le CAP marginal, ce qui correspond à un stock plus élevé. On retrouve ici l'idée des consignes de tir qui ont permis d'augmenter certaines populations (cervidé, sanglier, lièvre, etc.). L'externalité marginale de stock à l'optimum donne le prix de la bague ou du bracelet qu'il faudrait faire payer par pièce de gibier prélevé, pour que les chasseurs atteignent spontanément l'optimum. Le raisonnement est le même lorsqu' on considère la demande de sorties de chasse et non plus la demande de gibier. Une externalité marginale d'encombrement apparaît, et s'interprète comme la perte de bien-être imposée à tous les chasseurs du fait de l'augmentation marginale de la fréquentation. La fréquentation optimale est celle qui égalise le CAP pour la dernière journée de chasse à l'externalité marginale d'encombrement. Cette externalité donne le prix de la carte à la journée qui permettrait d'atteindre spontanément l'optimum. 


\section{Optimum collectif avec dégâts de gibier}

Le modèle précédent s'applique à des ressources cynégétiques qui ne créent pas de dommages externes. Dans le cas des cervidés et du sanglier, responsables notamment de dégâts aux cultures et de collisions automobiles, la valeur collective à maximiser est égale au $\mathrm{CAP}$ collectif des chasseurs diminuée des dommages externes, qui sont généralement fonction du stock de gibier. À l'optimum, le CAP marginal doit être égal à l'externalité marginale de stock, diminuée cette fois du dommage externe marginal, ce qui a pour effet d'augmenter le prélèvement et de diminuer le stock. Le stock optimal peut alors se rapprocher de celui de l'accès libre, qui peut donc être une méthode de gestion rationnelle pour ce type de gibier. Le dommage externe marginal à l'optimum donne la taxe par unité de stock qui décentralise l'optimum, dans le cas où les chasseurs limitent leurs prélèvements. Une autre voie pour restaurer l'optimum est de faire payer le dommage externe aux chasseurs, à la façon du principe pollueur-payeur. Leur profit est alors confondu avec la valeur collective ou sociale, ce qui explique que leur choix soit le même que celui du décideur public. Autrement dit, les chasseurs ne veulent plus augmenter le stock si le bénéfice marginal qu'ils en retirent est inférieur au dommage externe marginal qu'ils doivent désormais payer.

\section{Optimum environnemental}

En raison de l'impact majeur de leur activité sur les écosystèmes cynégétiques, les agriculteurs (et les exploitants forestiers) sont des offreurs potentiels d'améliorations environnementales pour le monde de la chasse (création de haies, cultures à gibier, jachère faune sauvage, réhabilitation et entretien des zones humides, réduction des pesticides, etc.). Pour modéliser le cas de l'amélioration environnementale, on maximise la valeur collective égale à la différence entre le CAP collectif et les coûts de l'amé- lioration, tous deux étant fonction du niveau de l'amélioration. À l'optimum, la somme des CAP marginaux des chasseurs pour bénéficier de l'amélioration doit être égale au coût marginal de l'amélioration, condition d'optimalité classique pour la fourniture des biens publics. Ici également cet optimum peut être atteint en faisant supporter par les chasseurs le coût de l'amélioration, c'est-àdire en appliquant le principe bénéficiairepayeur.

Ces optima ne sont généralement pas observés en raison des défaillances du marché, sauf peut-être dans le cas du propriétaire unique qui consomme ou loue sa chasse pourvue en gibier sédentaire et supporte l'intégralité du coût des aménagements et des dégâts (cas de certaines forêts privées à cervidés). C'est pourquoi les politiques cynégétiques, que nous allons analyser maintenant, devraient avoir pour objectif de s'en rapprocher.

\section{Analyse économique des politiques actuelles Des pistes d'amélioration}

Le rôle des politiques de la chasse consiste à internaliser les effets externes de stock, d'encombrement et environnementaux, en créant ou en appliquant mieux des droits de propriété et/ou en introduisant des mécanismes incitatifs qui vont assurer la coordination entre l'offre et la demande. Comme dans toute politique, on dispose de deux catégories d'instruments : les instruments quantitatifs de type quota et les instruments économiques de type taxe ou prix. Chaque catégorie possède ses avantages et ses inconvénients. Les quotas permettent de fixer des objectifs environnementaux et sociaux, mais ne sont pas incitatifs et ne permettent pas d'allouer les ressources de manière économiquement efficace, c'est-à-dire en maximisant la valeur ou en minimisant les coûts. En revanche, les instruments économiques sont incitatifs et économiquement efficaces, 
mais il est difficile de prédire leurs résultats environnementaux puisqu'on ne connaît pas les fonctions de réaction des agents (offre et demande). Lorsque les quotas ou les droits de propriété sont négociables sur un marché, ils cumulent les avantages des deux catégories, mais présentent des risques d'exclusion si le décideur public n'encadre pas les échanges. Des quotas de gibier individuels transférables seraient probablement mal adaptés au monde de la chasse, en revanche la mise en adjudication des droits de chasse par l'ONF et les communes d'Alsace-Moselle constitue un exemple de ces marchés encadrés par le décideur public.

\section{Gestion des prélèvements}

Le plan de chasse est l'instrument principal de gestion des populations de gibier. Introduit en 1963 pour certaines espèces de grand gibier à l'exception du sanglier, le plan de chasse a été progressivement généralisé à l'ensemble du territoire national et étendu au sanglier et au petit gibier sédentaire comme le lièvre et la perdrix (Charlez, 2008a). Il prévoit un nombre minimum et maximum d'animaux à prélever, de façon à concilier les intérêts agricoles, sylvicoles et cynégétiques. On retrouve ici la notion d'optimum social. Des taxes parafiscales sont perçues par animal à tirer (prix des bracelets cervidés ou boutons sanglier). Ces taxes servent à financer l'indemnisation des dégâts aux cultures et ne constituent pas un signal économique visant à internaliser les coûts externes. Il est d'ailleurs difficilement envisageable de gérer les prélèvements exclusivement par les prix, en raison de la méconnaissance de la demande et du caractère souvent collectif de la chasse, à quoi il faut ajouter l'acceptabilité par les chasseurs et les questions d'équité.

\section{Gestion de l'encombrement}

En revanche, les instruments économiques pourraient être explorés pour la gestion de l'encombrement en régime associatif. Dans le système actuel, les cotisations et timbres fiscaux sont forfaitaires et donnent généralement le droit de chasser à l'année. On a donc affaire à un coût fixe, qui agit sur le nombre de chasseurs ${ }^{10}$, mais n'est pas très limitant car il reste modéré. Pour réguler la pression de chasse, les associations ont recours au quota de jours, par exemple un ou deux jours fixes par semaine. Ce mode de gestion a l'inconvénient de concentrer les chasseurs, qui n'ont pas d'incitation à restreindre leur participation, sauf par annulation de leur CAP marginal. La qualité de la chasse s'en ressent, car les externalités d'encombrement font chuter le bien-être individuel et collectif des chasseurs ${ }^{11}$. Seul un coût variable en fonction de la fréquentation individuelle permettrait de limiter l'effort individuel et collectif. C'est pourquoi les cartes à la journée plutôt qu'à l'année sont un bon moyen de réguler l'encombrement. En outre, le tarif permet d'allouer davantage de sorties à ceux qui les valorisent le plus, ce qui contribue à augmenter le bien-être collectif. Les problèmes d'équité pourraient être traités en différenciant les tarifs, au minimum entre locaux et extérieurs, et en combinant quotas et tarifs (attribution d'un nombre limité de journées gratuites avec la carte de société et paiement à la marge des journées supplémentaires). En cas d'adjudication, le nombre de fusils est généralement limité, ce qui constitue une régulation de l'effort par l'exclusion, moins équitable et peut-être moins efficace que la carte à la journée.

\section{Gestion des dégâts}

En matière de gestion des dégâts, la plupart des Fédérations départementales des chasseurs (FDC) ont pratiqué jusqu'à une date récente la mutualisation des dépenses

10. Un chasseur prend un permis seulement si son espérance de bien-être est supérieure au prix du permis, ce qui élimine les chasseurs occasionnels qui pourraient être intéressés par une carte à la journée. 11. Cette pratique décourage les chasseurs extérieurs, notamment les citadins, qui doivent supporter un coût de déplacement avant de chasser. 
d'indemnisation. Chaque FDC gère un compte d'indemnisation qui finance les indemnisations ainsi que les frais d'estimation et de secrétariat. Il est alimenté par le produit des taxes des plans de chasse et des participations éventuelles des chasseurs de grand gibier du département (ONCFS, 2003). Ce système ne responsabilise pas les gestionnaires de territoires, qui ont intérêt à conserver une population qui maximise leur bien-être collectif. C'est pourquoi certaines FDC modulent le prix des bracelets par unité de gestion, en fonction des indemnisations versées (Klein et al., 2004). Cependant, la demande de plan de chasse étant faite par le détenteur du droit de chasse (Charlez, 2008a), un prix de bracelet élevé peut inciter à minorer le prélèvement minimum et aller ainsi à l'encontre de l'objectif poursuivi ${ }^{12}$. Belloy (2007) explique comment la FDC d'Indre et Loire répartit entre territoires le déficit entre recettes et dépenses d'indemnisation à l'intérieur d'un sous-massif soumis à plan de chasse, en faisant en sorte que chaque espèce paie ses dégâts et au prorata des hectares boisés pour la part sanglier. Une application plus complète du principe pollueur-payeur consisterait à répartir l'intégralité des dépenses d'indemnisation en diminuant le plus possible la taille du sous massif. Des dispositifs de ce type incitent les détenteurs du droit de chasse au gros gibier à trouver le bon compromis entre la qualité du loisir et les dégâts agricoles et forestiers. Ce système remplacerait avantageusement le prélèvement minimum et le bracelet payant, dont on a vu qu'ils étaient antagonistes, mais il faudrait maintenir le prélèvement maximum du plan de chasse pour éviter la tragédie des communs. En revanche, l'indemnisation par le détenteur du droit de chasse des dommages résultant des collisions automobiles n'est pas prévue. Une telle indemni-

12. La théorie montre que le bracelet payant internalise l'effet externe de stock en réduisant le prélèvement. sation se heurte à des problèmes juridiques qui rendent difficile son application, d'où le recours au fonds de garantie des assurances obligatoires de dommages (Charlez, 2004).

\section{Gestion de l'environnement}

Sur la question des aménagements agricoles ou forestiers favorables au gibier et à son habitat, la chasse associative est confrontée aux externalités qu'elle a générées. Il est donc logique qu'elle tente d'agir via les politiques publiques, parmi lesquelles il faut citer particulièrement les mesures d'accompagnement de la politique agricole commune (PAC). C'est ainsi que la jachère « environnement-faune sauvage » a été mise en place par la FNC avec le soutien des ministères de l'Agriculture et de l'Environnement et l'accord de la Commission européenne, en s'appuyant sur l'obligation de gel des terres imposée par la PAC. Les superficies consacrées à cette mesure ont atteint 30000 hectares, soit une faible proportion des territoires gérés par les associations. Elles ont chuté de $30 \%$ depuis la récente suppression de la jachère obligatoire, pour laquelle les chasseurs demandent des mesures compensatoires ${ }^{13}$. La prise en compte de la biodiversité dans l'éco-conditionnalité pourrait être une de ces mesures, mais il paraît difficile de l'imposer compte tenu de la redistribution des droits de propriété qu'elle implique. La proposition de la FNC de consacrer $5 \%$ de la SAU à la biodiversité pourrait d'ailleurs être déclinée sous forme de marché d'avantages écologiques : la norme de $5 \%$ pourrait être rendue échangeable sous réserve de cohérence écologique, en ce sens que certains agriculteurs pourraient acheter à d'autres l'obligation de consacrer tout ou partie de la norme de surface à la biodiversité. Un tel marché serait plus incitatif et plus efficace économiquement que la simple norme, car la

13. Communiqué de presse de la FNC du 10 octobre 2008 intitulé « Jachère et faune sauvage : qu'elles meurent ». 
production globale de biodiversité serait atteinte au moindre coût par les agriculteurs ayant les coûts les plus faibles.

Une norme sur la biodiversité produite par l'agriculture, avec ou sans compensation financière par l'État, est sans doute légitime car la biodiversité est un bien public qui ne profite pas qu'aux seuls chasseurs. Cependant, il est également légitime que le monde de la chasse, usager direct de la biodiversité, réfléchisse à l'application du principe bénéficiaire-payeur, comme moyen d'internaliser cette externalité. À cet égard, la tarification journalière évoquée précédemment permettrait de procurer des ressources pour la négociation des services environnementaux avec les agriculteurs et de gérer l'encombrement, tout en conservant l'équité dans l'accès. Le régime de droit local d'Alsace-Moselle et les forêts domaniales gérées par l'ONF fournissent d'ores et déjà des exemples de négociation entre propriétaires et chasseurs, susceptible d'inciter à des améliorations environnementales favorables au gibier. Ici, la coexistence de la décision publique et du marché devrait permettre de concilier gestion collective, efficacité économique et équité. C'est pourquoi il serait particulièrement intéressant d'analyser ces expériences sous l'angle cynégétique et économique.

\section{Conclusion}

Pour l'économiste, les situations où on observe une dégradation des ressources en gibier, de leurs habitats et de la qualité du loisir chasse s'expliquent par l'existence de biens collectifs et d'externalités, pour lesquels il n'y a pas de coordination entre l'offre et la demande de chasse. Ces défaillances du marché conduisent à une réduction du bien-être des chasseurs et c'est l'objectif des politiques cynégétiques que d'y pallier.

Le monde de la chasse a commencé à réfléchir aux mécanismes incitatifs qui permettraient d'internaliser les effets externes, par exemple sur la question des dégâts du gibier à l'agriculture, en les faisant payer aux gestionnaires de territoires, de façon à les responsabiliser. Cette démarche pourrait être élargie à d'autres effets externes, comme l'encombrement et l'amélioration des habitats du gibier, en étudiant dans quelle mesure le principe bénéficiairepayeur pourrait être mis en œuvre, sans rompre avec la tradition d'équité dans l'accès qui existe dans les associations.

Cette étude s'est efforcée de montrer en quoi les théories économiques de l'environnement et des ressources naturelles sont fécondes pour comprendre l'origine des problèmes qui frappent la chasse et $\mathrm{y}$ apporter des solutions. C'est là que réside son originalité, car cette analyse n'avait pas été conduite en France. C'est dans cet esprit que l'article propose d'appliquer à la chasse les deux solutions classiques d'internalisation, basées d'une part sur l'intervention centralisée (principes pollueur-payeur et bénéficiaire-payeur, quotas) et d'autre part sur l'application de droits de propriété (adjudication, négociation des droits de chasse, norme de biodiversité échangeable).

Une troisième solution a été proposée par Ostrom (1990), qui consiste à créer les conditions d'une coopération collective entre les propres utilisateurs de la ressource, dans l'objectif de résoudre le dilemme du prisonnier et le syndrome du passager clandestin. Cette solution paraît adaptée à l'organisation institutionnelle de la chasse en France, dans la mesure où la structuration en associations de chasse constitue autant de lieux propices pour conduire cette coopération. On peut penser que la réussite spectaculaire du plan de chasse de certaines espèces depuis 1990 (sanglier, cervidé, lièvre, notamment) est due à la conjonction de l'intervention centralisée et de la coopération à l'intérieur des associations de chasse, notamment pour faire adopter des consignes de tir spécifiques. La création de réserves de chasse ou la protection de zones de reproduction 
participent également de cette logique de coopération collective. En revanche, le constat de l'introduction semble pointer les limites de la coopération, interne ou externe, en tant qu'outil de gestion de l'encombrement, des dégâts de gibier et des habitats, notamment agricoles. À cet égard, il serait intéressant d'analyser les tentatives de coopération qui se sont tenues entre chasseurs et agriculteurs, au niveau local, régional ou national, à propos de dégâts et des habitats du gibier. Essayer de comprendre les raisons du succès ou de l'échec de la coopération, à la manière d'Ostrom, c'est-à-dire en analysant des expériences empiriques, constituerait un programme de recherches à part entière, inédit en France.

La question du coût de contrôle de la chasse n'a pas été abordée dans l'article. Ces coûts de contrôle sont assimilables à des coûts de transaction, dont on sait depuis Coase (1960) qu'ils conditionnent le choix entre des solutions centralisées ou de marché et plus généralement la nature des politiques environnementales (voir notamment Vatn et $a l .$, 1997). Les coûts de transaction ne sont pas seulement des freins au fonctionnement spontané des marchés, ils peuvent également enlever tout intérêt aux politiques publiques qui visent l'optimum de premier rang. À cet égard, l'existence d'un marché des chasses privées en forêt ou en plaine, ainsi que les systèmes d'adjudication observés en Alsace-Moselle et à l'ONF, semblent indiquer que les coûts de transaction liés à la marchandisation des droits de chasse ne seraient pas trop importants. Pour revenir à notre article, il conviendrait également de voir si les coûts de transaction associés aux mesures de gestion proposées (différentiation des dépenses d'indemnisation des dégâts, tarification à la journée, norme de biodiversité échangeable, négociation des services environnementaux) ne seraient pas supérieurs aux gains de bien-être collectif apportés par ces mesures. Il apparaît ici que la mesure de ces coûts de contrôle et de mise en œuvre devrait accompagner tout travail empirique sur l'analyse économique de la chasse.

Dans le souci d'augmenter le bien-être des chasseurs et de créer de la richesse dont pourraient bénéficier les acteurs des territoires ruraux, les politiques cynégétiques gagneraient à s'appuyer sur l'analyse économique. Pour fonder les analyses coûts-avantages et la récupération des coûts, les institutions qui sont chargées de gérer la chasse ont besoin de connaître l'offre et la demande de chasse, à l'instar de ce que recommande pour l'eau la directive cadre européenne (notamment dans son annexe 3 : «Analyse économique des utilisations de l'eau »). Il reviendrait aux recherches micro-économiques de mesurer d'une part le coût des aménagements agricoles et forestiers, sans oublier celui des dégâts commis par le gros gibier, et d'autre part le CAP pour prélever du gibier et bénéficier de populations abondantes, mais également le CAP des autres usagers de la faune sauvage pour pratiquer leurs activités ${ }^{14}$. Ces recherches sont aujourd'hui très embryonnaires en France, alors que ces questions sont étudiées de longue date dans les pays anglo-saxons. C'est pourquoi il convient de les développer en France, en complément des recherches biologiques sur la dynamique des populations de gibier et les capacités d'accueil des territoires.

14. Ce CAP peut donner une idée de l'importance économique relative des différents usages de la faune sauvage et aider ainsi à réaliser des arbitrages, qui concernent par exemple les forêts domaniales. Cependant, s'agissant de catégories sociales potentiellement différentes (différences de revenu notamment), il sera difficile de le comparer à celui des chasseurs. Par ailleurs, comme on a affaire à des usages gratuits sans prélèvement, la problématique n'est pas tant celle de la tarification publique ou du marché, dans laquelle on a besoin de la fonction de demande, que celle du partage de l'espace public et surtout de la durée d'usage (répartition des périodes de l'année et des jours de la semaine entre les différents usages). Notons cependant que la tarification des espaces récréatifs existe à l'étranger et se développe chez nous sous forme de parkings payants. 


\section{RÉFÉRENCES BIBLIOGRAPHIQUES}

Belloy A. (2007). Les modalités du financement de l'indemnisation des dégâts de grand gibier : de la mutualisation à la responsabilisation. Exemple de la FDC d'Indre et Loire. Colloque sur les modalités de gestion du sanglier, Fédération Nationale des Chasseurs, Office National de la Chasse et de la Faune Sauvage, Reims, $1^{\text {er }}$ et 2 mars.

Charlez A. (2003). Le propriétaire et son droit de chasse et de non chasse. Office National de la Chasse et de la Faune Sauvage.webmaster@oncfs.gouv.fr

Charlez A. (2004). Grand gibier et collisions automobiles. Faune sauvage, $\mathrm{n}^{\circ} 263$, p. 40-43.

Charlez A. (2008a). Plan de chasse et dégâts à la forêt : l'évolution. Faune sauvage, $\mathrm{n}^{\circ} 281, \mathrm{p} .56-63$.

Charlez A. (2008b). La loi sur les ACCA validée à trois reprises. Faune sauvage, $\mathrm{n}^{\circ} 280$, p. 51-55.

Clark C.-W. (1990). Mathematical bioeconomics: the optimal management of renewable resources. New York, John Wiley and Sons.

Coase R. (1960). The Problem of Social Cost. Journal of law and economics, vol. $3, \mathrm{n}^{\circ} 1$, p. 1-44.

Fischer B. (1999). La valeur du droit de chasse. Études foncières, $\mathrm{n}^{\circ}$ 82, p. 29-32.

FNC (2008). Livre blanc de la Fédération Nationale des Chasseurs pour le bilan de santé de la Politique Agricole Commune. Issy-les-Moulineaux, Fédération Nationale des Chasseurs.

Gordon H.-S. (1954). The economic theory of a common property resource: the fishery. Journal of political economy, $\mathrm{n}^{\circ}$ 62, p. 124-142.

Horan R.-D., Bulte E.-H. (2004). “Optimal and open access harvesting of multi-use species in a second best world". Environmental and Resource Economics, vol. 28, $\mathrm{n}^{\circ} 3$, p. 251-272.
Klein F., Baubet E., Toigo C., Leduc D., Saint-Andrieux C., Said S., Frechard C., Vallance M. (2004). La gestion $d u$ sanglier, des pistes et des outils pour réduire les populations. Technique et faune sauvage, Office National de la Chasse et de la Faune Sauvage, 32 p.

Landelle P. (2005). L'évolution des statuts juridiques de la faune sauvage en France. Faune sauvage, $\mathrm{n}^{\circ} 268$, p. 57-60.

ONCFS (2003). L'indemnisation des dégâts de grand gibier. Office National de la Chasse et de la Faune Sauvage, webmaster@oncfs.gouv.fr

Ostrom E. (1990) Governing the commons: The evolution of institutions for collective action. Cambridge University Press, $280 \mathrm{p}$.

Rakotoarison H., Point P. (2009). Une analyse coût-bénéfice du grand gibier : cas du sanglier dans la région Aquitaine. Colloque SFER «Chasse, territoires et développement durable : outils d'analyse, enjeux et perspectives », 2527 mars, Clermont-Ferrand.

Rakotoarison H., Point P., Malfait J.-J. (2009). Modélisation de l'impact économique de l'abroutissement par les chevreuils sur les forêts des Landes de Gascogne. Colloque SFER «Chasse, territoires et développement durable: outils d'analyse, enjeux et perspectives », 25-27 mars, Clermont-Ferrand.

Reitz F. (2006). Repeuplement en gibier sédentaire de plaine : une ancienne problématique toujours d'actualité. Faune sauvage, 274, 4-5.

Ropars C, Le Goffe P. (2009). La gestion du sanglier : modèle bio-économique, dégâts agricoles et prix des chasses en forêt. Colloque SFER «Chasse, territoires et développement durable: outils d'analyse, enjeux et perspectives », 25-27 mars, Clermont-Ferrand. 
RECHERCHES

Philippe LE GOFFE

Rondeau D. (2001). "Along the way back from the brink". Journal of Environmental Economics and Management, 42 (1), p. 156-182.

Skonhoft A., Olaussen J.-O. (2005) Managing a migratory species that is both a value and a pest. Land Economics 81(1), p. 34-50.

Vallance M., Arnauduc J.-P., Migot P. (2008). Atlas de la biodiversité de la faune sauvage, les 90 espèces chassables : Tout le gibier de France. Fédération Nationale des Chasseurs et Office National de la Chasse et de la
Faune Sauvage, Paris, Hachette, 503 p. Vatn A., Bakken L.-R., Lundeby H., Romstad E., Rrstad P.-K., Vold A., Botterweg P. (1997). Regulating nonpoint-source pollution from agriculture: an integrated modelling analysis. European Review of Agricultural Economics 24(2): 207-229. Zivin J., Hueth B.-M., Zilberman D. (2000). "Managing a multiple-use resource: the case of feral pigs in California rangeland". Journal of Environmental Economics and Management, 39(1), p. 189-204. 